\title{
Large-margin Predictive Latent Subspace Learning for Multi-view Data Analysis
}

\author{
Ning Chen ${ }^{\dagger}$, Jun Zhu ${ }^{\dagger}$, Member, IEEE, Fuchun Sun and Eric P. Xing, Senior Member, IEEE
}

\begin{abstract}
Learning salient representations of multi-view data is an essential step in many applications such as image classification, retrieval and annotation. Standard predictive methods, such as support vector machines, often directly use all the features available without taking into consideration the presence of distinct views and the resultant view dependencies, coherence, and complementarity that offer key insights to the semantics of the data, and are therefore offering weak performance and are incapable of supporting view-level analysis. This paper presents a statistical method to learn a predictive subspace representation underlying multiple views, leveraging both multi-view dependencies and availability of supervising side-information. Our approach is based on a multi-view latent subspace Markov network (MN) which fulfills a weak conditional independence assumption that multi-view observations and response variables are conditionally independent given a set of latent variables. To learn the latent subspace MN, we develop a large-margin approach which jointly maximizes data likelihood and minimizes a prediction loss on training data. Learning and inference are efficiently done with a contrastive divergence method. Finally, we extensively evaluate the large-margin latent MN on real image and hotel review datasets for classification, regression, image annotation and retrieval. Our results demonstrate that the large-margin approach can achieve significant improvements in terms of prediction performance and discovering predictive latent subspace representations.
\end{abstract}

Index Terms - Latent subspace model, Large-margin learning, Classification, Regression, Image retrieval and annotation.

\section{INTRODUCTION}

$\mathrm{M}$ ODERN data analytic problems in social media, information technology, and natural sciences often involve rich data consisting of multiple information modalities. For example, in a moment-sharing social network such as Instagram, a photo record would include image, text (status updates and viewer opinions), and various meta information such as user demographics, geo-tags, time stamps, etc.; in a biomedical data repository, a clinical sample record may include gene expression intensity, protein activity status, clinical traits, and patient information with family history. These different modalities represent different angles to reveal the fundamental characteristics and properties of the study subjects, and is often referred as views of the subjects.

Proper integration of multiple views present in multimodal data is of paramount importance for seeking accurate distillation of salient semantic representations of the study objects, therefore numerous efforts along this direction can be found in the literature. To name a few, [6] studied co-training scheme of a classification model for web pages based on both content and link anchor text; [44] proposed a dual view latent space model for video shot based on both color/shape of the keyframe and the

- + indicates equal contributions from these authors.

- Ning Chen, Jun Zhu and Fuchun Sun: Dept. of CS \& T, TNList Lab, State Key Lab of ITS, Tsinghua University, Beijing 100084 China. \{chenn07@mails,dcszj@,fcsun@\}.tsinghua.edu.cn

- Jun Zhu was with School of Computer Science, Carnegie Mellon University, Pittsburgh, PA 15213, USA.

- Eric P. Xing: School of Computer Science, Carnegie Mellon University, Pittsburgh, PA 15213, USA. epxing@cs.cmu.edu corresponding closed captions; and this list continues to grow, under various contexts and addressing a diverse range of data forms [17][11][34][35][14]. However, most of these approaches for multi-view integration and distillation do not go hand-in-hand with main stream predictive methods such as support vector machines (SVMs) [8] or Boosting algorithms [19] to form a unified system that allows strongly predictive latent semantic representations of multi-view data to be extracted. Typically, standard predictive methods would use one of the following strategies: 1) build a single classifier on observed features from all views, without taking into consideration the presence of distinct views; 2) build a set of classifiers defined on each view, regardless of the relationships among views; and 3) let a latent space model such as a multi-view topic model to distill the latent representations of data without considering the predictive information ${ }^{1}$, and then apply a downstream classifier on such representations [44]. While offering many insights on how multi-view data can be worked with, these approaches appear to enjoy limited practical benefits from the extra information present in multi-view data, in terms of predictive performance [7], computational cost [35], and power for view-level analysis [14] such as predicting tags for image annotation or analyzing the underlying relationships among views.

Moreover, with the rapid increase of free on-line information such as user tagging, ratings, etc., various forms of side-information that can potentially offer "free" supervision over the media data have led to a need for new models and training schemes that can make effective use

1. But see [45] for a few exception with limited performance gain. 
of such information to achieve better results, such as more discriminative latent representations of image contents and more accurate image classifiers. In this paper, we develop a new statistical framework that enables one to learn a predictive latent space representation shared by multi-view data by leveraging supervising side information; and to perform both view-level analysis (e.g., image annotation) and response-level predictions (e.g., classification) based on the learned representation.

Our method builds on a probabilistic latent subspace model that relates features of perceivable entities (e.g., images) to abstract concepts (e.g., latent topics) in a probabilistic way, which allows flexible and efficient statistical reasoning and inference. Our model is a generic multi-view latent space Markov network (MN) that builds on a weak conditional independence assumption that the data from different views and the response variables are conditionally independent given a set of latent variables. This conditional independence is weaker than the typical assumption (e.g., in the seminal work of co-training [6]) that multi-view data are conditionally independent given the very low dimensional response variables [18]. Although in principle a directed Bayesian network (BN) (e.g., latent Dirichlet allocation (LDA) [5][4][48]) can be extended to handle multi-view data, its conditional dependency properties could make it hard to perform posterior inference because all latent variables are coupled given observed variables [41]. In contrast, undirected latent variable models, such as ours and those presented in [41][32][44], could be very efficient in inference because of the conditional independence.

One critical limitation of existing paradigms for learning probabilistic latent subspace models, as discussed in [48], is that the commonly used likelihood-based methods are often not discriminative enough to leverage the supervising side information accompanying the multi-view data to extract a strongly predictive representation; and is prone to undesirable effects such as over-fitting to small data [41][44][45][28]. To overcome such limitations, we use a completely different and arguably more desirable learning paradigm based on the maximum margin principle. More specifically, we develop a new discriminative learning approach for the proposed latent space Markov network, which jointly maximizes the likelihood of multi-view data and minimizes a prediction loss on the labels from side information (e.g., hinge-loss for classification or $\epsilon$-insensitive loss [33] for regression) to discover a strongly predictive subspace representation and learn a prediction model thereupon. The learning and inference problems are efficiently solved with an extension of the contrastive divergence method [40]. Extensive experiments show that the proposed large-margin approach can achieve significant improvements in terms of prediction performance and semantic saliency of the predictive latent subspace representations. Moreover, the inference in the latent space $\mathrm{MN}$ is much faster and easier compared with the directed counterpart models, e.g., MedLDA [48].
The remaining paper is organized as follows. Section 2 reviews related work. Section 3 presents the multi-view latent subspace MN. Section 4 presents the large-margin training methods for both classification and regression. Section 5 presents extensive empirical evaluation on various datasets. Finally, Section 6 concludes with future research directions discussed.

\section{Related Work}

The literature of discovering latent representations from large collections of data consists of both deterministic (e.g., canonical correlation analysis (CCA) [24][26][1] and Fisher discriminant analysis (FDA) [15]) and probabilistic (e.g., directed LDA [5][39][50] and undirected Harmoniums [41][32][44]) methods. A deterministic method cannot be easily extended to perform view-level predictions, such as image annotation, and it would also need a density estimator in order to apply the information criterion [11] to detect view disagreement. Thus, we choose the probabilistic framework and base our approach on an undirected multi-view latent space model, which enjoys nice properties as discussed.

To consider supervising side information, supervised latent space models have been developed, including supervised LDA [4][39][48] and supervised Harmoniums [45][28]. However, almost all these models are learned using likelihood-based estimation, which often involves dealing with an intractable normalization factor [39][50] and may not yield improvements compared with the standard prediction tools based on purely discriminative ideas (e.g., SVM) [45]. The recent work of MedLDA [48] has shown a promising direction of applying the largemargin principle to learn predictive latent space representations which could be more suitable for prediction (e.g., classification). Other developments along this line include the large-margin upstream scene understanding models [49] and the conditional topic models with features [50]. However, these methods are all directed Bayesian networks, which may involve a hard inference problem, as we have discussed. The present work represents an important contribution of deploying the largemargin principle to learn undirected latent space models.

The large-margin principle has also been applied to learn Markov networks with latent variables [51][16][46]. However, their goals are mainly to use latent variables to capture residual and high-order dependency for improving prediction performance, essentially different from ours of learning predictive latent representations of the data. Our approach is also different from the existing much research that has been done on exploring multiview information to alleviate semi-supervised learning [6][14][2][18][26], unsupervised clustering [9] and structured output problems [21]. Other work that relates to ours includes the hybrid generative/discriminative learning [31], which uses likelihood-based estimation, and the sufficient dimensionality reduction method$s$ [20]. Finally, this paper is a systematic extension of the preliminary conference version [10]. 


\section{Multi-View Latent Subspace MNS}

In this section, we present a multi-view latent subspace Markov network (MN) by incorporating complex structures on each view. We will start with an unsupervised latent subspace $\mathrm{MN}$ and then present a supervised latent subspace MN based on maximum likelihood estimation.

\subsection{Unsupervised Multi-view Latent Subspace MNs}

Fig. 1 shows the structure of a two-view latent subspace MN which consists of two types of input data $\mathbf{X} \triangleq\left\{X_{i}\right\}_{i=1}^{N}$ and $\mathbf{Z} \triangleq\left\{Z_{j}\right\}_{j=1}^{M}$, each corresponding to a view; and a set of latent variables $\mathbf{H} \triangleq\left\{H_{k}\right\}_{k=1}^{K}$, corresponding to the latent representations one desires to infer. We encode the structure of the variables on each view using a Markov network. Purely for simplicity of $\mathrm{p}$ resentation, we focus on the case of pairwise interactions between variables within each view. We emphasize that our results extend easily to more general cases of higherorder dependencies. Let $E_{x}$ denote the set of edges ${ }^{2}$ between the input variables $\mathbf{X}$, and likewise for $E_{z}$. We will use $e$ to denote one individual edge and use $\mathbf{X}_{e}$ to denote the variables associated with $e$.

A constructive way to define the joint distribution of a latent subspace MN is as follows. First, we define the distribution of the data on each view and the latent variables separately. For each view, we use an exponential family distribution

$$
\begin{aligned}
& p(\mathbf{x})=r(\mathbf{x}) \exp \left\{\sum_{e \in E_{x}} \theta_{e}^{\top} \phi\left(\mathbf{x}_{e}\right)-A(\theta)\right\}, \\
& p(\mathbf{z})=s(\mathbf{z}) \exp \left\{\sum_{e \in E_{z}} \eta_{e}^{\top} \psi\left(\mathbf{z}_{e}\right)-B(\eta)\right\},
\end{aligned}
$$

where $\phi$ and $\psi$ are vectors of feature functions; $\theta$ and $\eta$ are weights; and $A$ and $B$ are $\log$ partition functions. Like [41], we will treat $\log (r(\mathbf{x}))$ and $\log (s(\mathbf{z}))$ as additional features multiplied by a constant. For the latent variables $\mathbf{H}$, each component $H_{k}$ has an exponential family distribution and

$$
p(\mathbf{h})=\prod_{k} p\left(h_{k}\right)=\prod_{k} \exp \left\{\lambda_{k}^{\top} \varphi\left(h_{k}\right)-C_{k}\left(\lambda_{k}\right)\right\}
$$

where $\varphi\left(h_{k}\right)$ is the vector of features of $h_{k} . C_{k}$ is another log-partition function.

Then, the joint model distribution is defined by combining the above components in the log-domain and introducing additional terms that couple the random variables $\mathbf{X}, \mathbf{Z}$ and $\mathbf{H}$. Specifically, we have

$$
\begin{array}{r}
p(\mathbf{x}, \mathbf{z}, \mathbf{h}) \propto \exp \left\{\sum_{e \in E_{x}} \theta_{e}^{\top} \phi\left(\mathbf{x}_{e}\right)+\sum_{e \in E_{z}} \eta_{e}^{\top} \psi\left(\mathbf{z}_{e}\right)+\sum_{k} \lambda_{k}^{\top} \varphi\left(h_{k}\right)\right. \\
\left.+\sum_{e \in E_{x}, k} \phi\left(\mathbf{x}_{e}\right)^{\top} \mathbf{W}_{e}^{k} \varphi\left(h_{k}\right)+\sum_{e \in E_{z}, k} \psi\left(\mathbf{z}_{e}\right)^{\top} \mathbf{U}_{e}^{k} \varphi\left(h_{k}\right)\right\},(2)
\end{array}
$$

where $\mathbf{W}$ and $\mathbf{U}$ are feature weights. From the joint distribution, we can derive the conditional distributions on each view with shifted parameters $(\hat{\theta}, \hat{\eta}, \hat{\lambda})$

$$
\begin{aligned}
p(\mathbf{x} \mid \mathbf{h}) & =\exp \left\{\sum_{e \in E_{x}} \hat{\theta}_{e}^{\top} \phi\left(\mathbf{x}_{e}\right)-A(\hat{\theta})\right\} \\
p(\mathbf{z} \mid \mathbf{h}) & =\exp \left\{\sum_{e \in E} \hat{\eta}_{e}^{\top} \psi\left(\mathbf{z}_{e}\right)-B(\hat{\eta})\right\} \\
p(\mathbf{h} \mid \mathbf{x}, \mathbf{z}) & =\prod_{k} \exp \left\{\hat{\lambda}_{k}^{\top} \varphi\left(h_{k}\right)-C_{k}\left(\hat{\lambda}_{k}\right)\right\},
\end{aligned}
$$

2. We treat a singleton vertex as a degenerate edge.

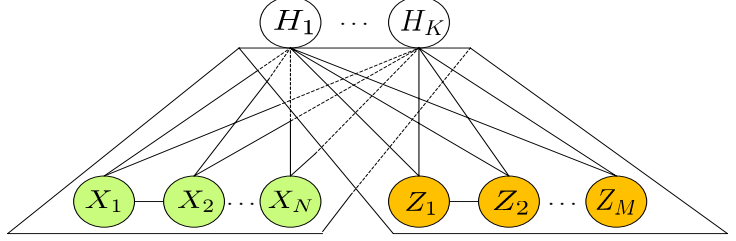

Fig. 1. An unsupervised two-view latent subspace MN.

where $\hat{\theta}_{e}=\theta_{e}+\sum_{k} \mathbf{W}_{e}^{k} \varphi\left(h_{k}\right), \hat{\eta}_{e}=\eta_{e}+\sum_{k} \mathbf{U}_{e}^{k} \varphi\left(h_{k}\right)$, and $\hat{\lambda}_{k}=\lambda_{k}+\left(\sum_{e \in E_{x}} \phi\left(\mathbf{x}_{e}\right)^{\top} \mathbf{W}_{e}^{k}+\sum_{e \in E_{z}} \psi\left(\mathbf{z}_{e}\right)^{\top} \mathbf{U}_{e}^{k}\right)^{\top}$. We can see that conditioned on the latent variables, both $p(\mathbf{x} \mid \mathbf{h})$ and $p(\mathbf{z} \mid \mathbf{h})$ define a Markov network, which is known as conditional random fields (CRFs) [27], where $\mathbf{h}$ correspond to global conditions and $\mathbf{x}$ or $\mathbf{z}$ correspond to structured prediction variables in CRFs.

Reversely, one can start with defining the local conditional distributions as above and directly write the compatible joint distribution, which is of the log-linear form as in Eq. (2). In the sequel, we use $\Theta$ to denote all the parameters $(\theta, \eta, \lambda, \mathbf{W}, \mathbf{U})$. It is worth noting that both the exponential family Harmonium (EFH) [41] and its extension of dual-wing Harmonium (DWH) [44] are special cases of multi-view latent subspace MNs, when the generalized edge sets $E_{x}$ and $E_{z}$ contain only singleton vertices. Therefore, it is not surprising to see that multi-view MNs inherit the widely advocated property of EFH that the model distribution can be constructively defined based on local conditionals on each view.

We briefly introduce DWH here as it sets up the ground for our experiments in Section 5. As in [44], DWH has a two-view structure, where $\mathbf{X}$ is a vector of discrete word features (e.g., image tags) and $\mathbf{Z}$ is a vector of real-valued features (e.g., color histograms). We assume that each $X_{i}$ is a Bernoulli variable that denotes whether the $i$ th term of a dictionary appears or not in an image, and each $Z_{j}$ is a real number that denotes the normalized color histogram of an image. Each realvalued $H_{k}$ follows a univariate Gaussian distribution. Therefore, the conditional distributions can be defined as

$$
\begin{aligned}
p\left(x_{i}=1 \mid \mathbf{h}\right) & =\operatorname{Logistic}\left(\alpha_{i}+\mathbf{W}_{i} \cdot \mathbf{h}\right), \\
p\left(z_{j} \mid \mathbf{h}\right) & =\mathcal{N}\left(z_{j} \mid \sigma_{j}^{2}\left(\beta_{j}+\mathbf{U}_{j} \cdot \mathbf{h}\right), \sigma_{j}^{2}\right), \\
p\left(h_{k} \mid \mathbf{x}, \mathbf{z}\right) & =\mathcal{N}\left(h_{k} \mid \mathbf{x}^{\top} \mathbf{W}_{\cdot k}+\mathbf{z}^{\top} \mathbf{U}_{\cdot k}, 1\right),
\end{aligned}
$$

where $\mathbf{W}_{i}$. and $\mathbf{W}_{. k}$ denote the $i$ th row and $k$ th column of $\mathbf{W}$, respectively. Likewise for $\mathbf{U}_{j}$. and $\mathbf{U}_{. k}$.

To learn the unsupervised multi-view latent subspace MNs, a natural method is the maximum likelihood estimation (MLE), which has been widely used to train directed [39][47] and undirected latent variable models [41][32][44][45]. To deal with the intractable loglikelihood $\log p(\mathbf{x}, \mathbf{z})$, an approximation method such as mean field or contrastive divergence [44] is usually applied. More details will be provided along with the algorithm development for large-margin learning.

To use the unsupervised multi-view MN for prediction (e.g., classification), a naïve method is a two-stage procedure: 1) using the latent subspace $\mathrm{MN}$ to discover latent representations; and 2) feeding the latent representations into a downstream prediction model (e.g., SVM). 
This two-step procedure can be rather sub-optimal for prediction because supervising information is ignored in discovering the latent representations. Moreover, as we have stated, supervising side information can be almost "free" to obtain; thus it is desirable to develop new models and learning methods to consider such information for improving performance. Below, we present supervised latent subspace MNs, which incorporate supervising side information into the procedure of discovering latent subspace representations. As we shall see, if learned appropriately, e.g., using large-margin training, a supervised latent subspace $\mathrm{MN}$ can achieve significant improvements in discovering predictive latent subspace representations and prediction performance.

\subsection{Supervised Multi-view Latent Subspace MNs}

Similar to learning an unsupervised latent subspace MN, MLE is the natural method to learn a supervised latent subspace MN. In this section, we present the MLE-based supervised latent subspace $\mathrm{MN}$, which would motivate our development of a large-margin approach.

In order to perform MLE, we need to define a likelihood model for observed data, including input features and response variables in the supervised case. Specifically, let $Y$ be the response variable and $\mathbf{V}$ be the parameters of a response variable model. Then we need to define the joint distribution $p(\mathbf{x}, \mathbf{z}, \mathbf{h}, y)$. We consider univariate prediction, where $Y$ can be a discrete variable for classification or a continuous variable for regression. Based on the constructive definition, we need to specify the conditional distribution of $Y$ given $\mathbf{H}$ in order to define $p(\mathbf{x}, \mathbf{z}, \mathbf{h}, y)$. For the general multi-class classification, where $y \in\{1, \cdots, T\}$, we define the conditional distribution using a softmax function ${ }^{3}$

$$
p(y \mid \mathbf{h})=\frac{\exp \left\{\mathbf{V}^{\top} \mathbf{f}(y, \mathbf{h})\right\}}{\sum_{y^{\prime}} \exp \left\{\mathbf{V}^{\top} \mathbf{f}\left(y^{\prime}, \mathbf{h}\right)\right\}},
$$

where $\mathbf{f}(y, \mathbf{h})$ is the feature vector whose elements from $(y-1) K+1$ to $y K$ are those of $\mathbf{h}$ and all others are $0 . \mathbf{V}$ is a stacking parameter vector of $T$ sub-vectors $\mathbf{V}_{y}$, of which each one corresponds to a class label $y$. Then, the joint distribution $p(\mathbf{x}, \mathbf{z}, \mathbf{h}, y)$ has the same form as in Eq. (2), but with an additional term of $\mathbf{V}^{\top} \mathbf{f}(y, \mathbf{h})=\mathbf{V}_{y}^{\top} \mathbf{h}$ in the exponential. For regression, where $y \in \mathbb{R}$, we define the conditional distribution as a normal distribution

$$
p(y \mid \mathbf{h})=\mathcal{N}\left(y \mid \mathbf{V}^{\top} \mathbf{h}, \sigma^{2}\right),
$$

where $\mathbf{V}$ is now a $K$-dim vector. Then, the joint distribution $p(\mathbf{x}, \mathbf{z}, \mathbf{h}, y)$ has the similar form as in Eq. (2) with an additional term of $-\frac{1}{2 \sigma^{2}}\left(y^{2}-y \mathbf{V}^{\top} \mathbf{h}\right)$ in the exponential.

Note that the supervised hierarchical (or tri-wing) Harmonium (TWH) [45] is a special case of the supervised latent subspace $\mathrm{MN}$ for classification. With the above joint likelihood function, we can perform standard

3. For notation simplicity, we omit the offset parameters in both classification and regression models. Offset parameters can be easily included by adding one unit dimension to $\mathbf{h}$.
MLE by using contrastive divergence or mean field approximation to learn the parameters ${ }^{4}$. The procedure is generally similar as that in learning TWH [45]. The major difference lies in posterior inference, which would be clear after we have presented the large-margin learning.

\section{LARGE-MARGIN SUPERVISED MULTI-VIEW LATENT SUBSPACE MNS}

As stated above, the MLE-based supervised latent subspace $\mathrm{MN}$ requires defining a normalized distribution as in Eq. (3), of which the normalization factor could make the inference hard, especially in directed models [39][50]. Moreover, as shown in [45] and our empirical studies, the MLE-based model may not obtain improvements over the naïve two-step method discussed at the end of Section 3.1. These motivate us to develop a more discriminative procedure for learning supervised latent subspace MNs. In this section, we present a large-margin supervised latent subspace $\mathrm{MN}$ for discovering predictive latent subspace representations from multi-view data by incorporating the widely available supervising side information, which can be discrete for classification or continuous for regression.

\subsection{Classification}

We first present the classification model. For brevity, we consider the general multi-class classification. The binary case can be similarly derived.

\subsubsection{Problem Definition}

Similar to the log-linear model in Eq. (3), we define the latent discriminant function $F(y, \mathbf{h} ; \mathbf{V})$ as linear when latent variables $\mathbf{H}$ are given, that is, $F(y, \mathbf{h} ; \mathbf{V})=$ $\mathbf{V}^{\top} \mathbf{f}(y, \mathbf{h})$, where $\mathbf{f}$ and $\mathbf{V}$ are defined the same as in Eq. (3). Now, the problem is how to consider the uncertainty of $\mathbf{H}$ in the deterministic large-margin principle. Here, we take the expectation (i.e., first moment) of the latent variables $\mathbf{H}$ and define the expected prediction rule

$$
\begin{aligned}
y^{*} & \triangleq \underset{y}{\arg \max } \mathbb{E}_{p(\mathbf{h} \mid \mathbf{x}, \mathbf{z})}[F(y, \mathbf{h} ; \mathbf{V})] \\
& =\underset{y}{\arg \max } \mathbf{V}^{\top} \mathbb{E}_{p(\mathbf{h} \mid \mathbf{x}, \mathbf{z})}[\mathbf{f}(y, \mathbf{h})]
\end{aligned}
$$

where the expectation can be efficiently computed with the factorized form of $p(\mathbf{h} \mid \mathbf{x}, \mathbf{z})$ when $\mathbf{x}$ and $\mathbf{z}$ are fully observed. If missing values exist in $\mathbf{x}$ or $\mathbf{z}$, an inference procedure is needed to compute the expectation of the missed components, as detailed below in Eq. (7).

Then, learning is to find an optimal $\mathbf{V}^{*}$ that minimizes a loss function. Here, we minimize the hinge loss, as used in the very successful large-margin SVMs. Specifically, given training data $\mathcal{D}=\left\{\left(\mathbf{x}_{d}, \mathbf{z}_{d}, y_{d}\right)\right\}_{d=1}^{D}$, the hinge loss of the expected predictive rule (5) is

$$
\mathcal{R}_{\text {hinge }}(\mathbf{V}) \triangleq \sum_{d} \max _{y}\left[\Delta \ell_{d}(y)-\mathbf{V}^{\top} \mathbb{E}_{p\left(\mathbf{h} \mid \mathbf{x}_{d}, \mathbf{z}_{d}\right)}\left[\Delta \mathbf{f}_{d}(y)\right]\right]
$$

4. A discriminative method that maximizes the conditional likelihood $p(y \mid \mathbf{x}, \mathbf{z})$ could be developed as in [28], but it could be inferior to a hybrid generative/discriminative method. 
where $\Delta \ell_{d}(y)$ is a loss function (e.g., 0/1-loss) that measures how different a candidate prediction $y$ is compared to the true label $y_{d}$, and $\mathbb{E}_{p\left(\mathbf{h} \mid \mathbf{x}_{d}, \mathbf{z}_{d}\right)}\left[\Delta \mathbf{f}_{d}(y)\right]=$ $\mathbb{E}_{p\left(\mathbf{h} \mid \mathbf{x}_{d}, \mathbf{z}_{d}\right)}\left[\mathbf{f}\left(y_{d}, \mathbf{h}\right)\right]-\mathbb{E}_{p\left(\mathbf{h} \mid \mathbf{x}_{d}, \mathbf{z}_{d}\right)}[\mathbf{f}(y, \mathbf{h})]$. It can be proved that the hinge loss is an upper bound of the empirical error $\mathcal{R}_{e m p} \triangleq \sum_{d} \Delta \ell_{d}\left(y_{d}^{*}\right)$. Applying the principle of regularized risk minimization, we define the joint problem of learning a prediction model $\mathbf{V}$ and a likelihood model $\Theta$ for fitting the input data as solving

$$
\mathrm{P} 1: \min _{\Theta, \mathbf{V}} L(\Theta)+\frac{1}{2} C_{1}\|\mathbf{V}\|_{2}^{2}+C_{2} \mathcal{R}_{\text {hinge }}(\mathbf{V})
$$

where $L(\Theta) \triangleq-\sum_{d} \log p\left(\mathbf{x}_{d}, \mathbf{z}_{d}\right)$ is the negative data likelihood and $C_{1}$ and $C_{2}$ are non-negative constants, which can be selected via cross-validation. Note that $\mathcal{R}_{\text {hinge }}$ is also a function of $\Theta$.

Since problem (6) jointly maximizes the data likelihood and minimizes a training loss, it can be expected that by solving this problem we can find a predictive latent subspace representation (i.e., $\mathbb{E}_{p(\mathbf{h} \mid \mathbf{x}, \mathbf{z})}[\mathbf{h}]$ ) and a prediction model (represented by the parameter $\mathbf{V}$ ), which on one hand tend to predict as accurate as possible on the training data, while on the other hand tend to explain the data well. More insights will be provided in the next section along with the algorithm development.

\subsubsection{Optimization with Contrastive Divergence}

Since the data likelihood $L(\Theta)$ is generally intractable to compute, we use an efficient variational inference method (i.e., contrastive divergence) [23][40][41][44] to approximate the joint likelihood. Specifically, we derive a variational approximation $\mathcal{L}^{v}\left(q_{0}, q_{1}\right)$ to represent the negative log-likelihood $L(\Theta)$ :

$$
\mathcal{L}^{v}\left(q_{0}, q_{1}\right) \triangleq R\left(q_{0}(\mathbf{x}, \mathbf{z}, \mathbf{h}), p(\mathbf{x}, \mathbf{z}, \mathbf{h})\right)-R\left(q_{1}(\mathbf{x}, \mathbf{z}, \mathbf{h}), p(\mathbf{x}, \mathbf{z}, \mathbf{h})\right),
$$

where $R(q, p)$ is the relative entropy between distributions $q$ and $p ; q_{0}$ is a variational distribution with $\mathbf{x}$ and $\mathbf{z}$ clamped to their observed values, while $q_{1}$ is a distribution with all the variables free. For $q$ (either $q_{0}$ or $\left.q_{1}\right)$, we employ the structured mean field assumption [43] that ${ }^{5} q(\mathbf{x}, \mathbf{z}, \mathbf{h})=q(\mathbf{x}) q(\mathbf{z}) q(\mathbf{h})$.

Substituting the variational approximation $\mathcal{L}^{v}\left(q_{0}, q_{1}\right)$ into problem (6), we get an approximate objective function $\mathcal{L}\left(\Theta, \mathbf{V}, q_{0}, q_{1}\right)$. Then, we can develop an alternating minimization method which iteratively minimizes $\mathcal{L}\left(\Theta, \mathbf{V}, q_{0}, q_{1}\right)$ over $\left(q_{0}, q_{1}\right)$ and $(\Theta, \mathbf{V})$. The problem of solving $q_{0}$ and $q_{1}$ is posterior inference. Specifically, for a variational distribution $q$ (can be $q_{0}$ or $\left.q_{1}\right)$, we keep $(\Theta, \mathbf{V})$ fixed and update each marginal as

$$
\begin{aligned}
& q(\mathbf{x})=p\left(\mathbf{x} \mid \mathbb{E}_{q(\mathbf{h})}[\mathbf{h}]\right), \quad q(\mathbf{z})=p\left(\mathbf{z} \mid \mathbb{E}_{q(\mathbf{h})}[\mathbf{h}]\right) \\
& q(\mathbf{h})=\prod_{k} p\left(h_{k} \mid \mathbb{E}_{q(\mathbf{x})}[\mathbf{x}], \mathbb{E}_{q(\mathbf{z})}[\mathbf{z}]\right)
\end{aligned}
$$

For $q_{0},(\mathbf{x}, \mathbf{z})$ are clamped at their observed values, and only $q_{0}(\mathbf{h})$ is updated, which can be very efficiently done due to its factorized form. The distribution $q_{1}$ is achieved

5. The parametric form assumptions of $q$, as employed in previous work [44][45], are not needed. by performing the above updates starting from $q_{0}$, and several iterations (e.g., 5 used in our experiments) can yield a good $q_{1}$. Note that Eq. (7) holds for exponential family models where $\mathbf{h}$ enters linearly in $\ln p(\mathbf{x}, \mathbf{z} \mid \mathbf{h})$. Please see [40] for more details. Again, we can observe that both $q(\mathbf{x})$ and $q(\mathbf{z})$ are CRFs, with the expectation of $\mathbf{H}$ as the condition. Therefore, for linear-chain models, we can use a message passing scheme [27] to infer their marginal distributions, as needed for parameter estimation and view-level prediction (e.g., image annotation), as we shall see. For generally structured models, approximate inference techniques [37] can be applied.

After we have inferred $q_{0}$ and $q_{1}$, parameter estimation can be solved with coordinate descent by alternating the following two steps: 1 ) estimating $\mathbf{V}$ with $\Theta$ fixed: this problem is learning a multi-class SVM [13], which can be efficiently done with existing solvers; and 2) estimating $\Theta$ with $\mathbf{V}$ fixed: this can be solved with sub-gradient descent. By defining $\Delta \mathbb{E}[\cdot] \triangleq \mathbb{E}_{q_{1}}[\cdot]-\mathbb{E}_{q_{0}}[\cdot]$, we can compute the sub-gradient as follows. For $\theta$, we have $\forall e \in E_{x}, \partial \theta_{e}=\Delta \mathbb{E}\left[\phi\left(\mathbf{x}_{e}\right)\right]$; for $\eta$, we have $\forall e \in E_{z}, \partial \eta_{e}=\Delta \mathbb{E}\left[\psi\left(\mathbf{z}_{e}\right)\right]$; for $\lambda$, we have $\forall k, \partial \lambda_{k}=\Delta \mathbb{E}\left[\varphi\left(h_{k}\right)\right] ;$ and for $\mathbf{W}$ and $\mathbf{U}$, we have:

$$
\begin{aligned}
& \partial \mathbf{W}_{e}^{k}=\Delta \mathbb{E}\left[\phi\left(\mathbf{x}_{e}\right) \varphi\left(h_{k}\right)^{\top}\right]-C_{2} \sum_{d}\left(\mathbf{V}_{y_{d} k}-\mathbf{V}_{\bar{y}_{d} k}\right) \frac{\partial \mathbb{E}_{q_{0}}\left[h_{k}\right]}{\partial \mathbf{W}_{e}^{k}} \\
& \partial \mathbf{U}_{e}^{k}=\Delta \mathbb{E}\left[\psi\left(\mathbf{z}_{e}\right) \varphi\left(h_{k}\right)^{\top}\right]-C_{2} \sum_{d}\left(\mathbf{V}_{y_{d} k}-\mathbf{V}_{\bar{y}_{d} k}\right) \frac{\partial \mathbb{E}_{q_{0}}\left[h_{k}\right]}{\partial \mathbf{U}_{e}^{k}},
\end{aligned}
$$

where $\bar{y}_{d}=\arg \max _{y}\left[\Delta \ell_{d}(y)+\mathbf{V}^{\top} \mathbb{E}_{q_{0}}[\mathbf{f}(y, \mathbf{h})]\right.$ is the loss-augmented prediction. The expectation $\mathbb{E}_{q_{0}}\left[\phi\left(\mathbf{x}_{e}\right)\right]$ is actually the count frequency of $\phi\left(\mathbf{x}_{e}\right)$ on the training data $\mathcal{D}$; likewise for $\mathbb{E}_{q_{0}}\left[\psi\left(\mathbf{z}_{e}\right)\right]$. With the above sub-gradients, we apply L-BFGS [29], which uses line search to choose a step size, to iteratively solve for the optimum $\Theta$ until convergence.

Note that in our integrated large-margin formulation, the sub-gradients corresponding to $\mathbf{W}$ and $\mathbf{U}$ contain an additional term (i.e., the third term) compared to the standard DWH [44] with contrastive divergence approximation. This additional term introduces a regularization effect to the latent subspace model. If the loss-augmented prediction $\bar{y}_{d}$ differs from the true label $y_{d}$, this term will be non-zero and it will bias the model toward discovering a better representation for prediction. As we shall see, this bias term will make the large-margin based multi-view latent subspace model tend to discover a latent representation that is more predictive.

\subsection{Regression}

In this section, we present the large-margin latent subspace $\mathrm{MN}$ for regression.

\subsubsection{Problem Definition}

Similar to the classification model, we define the linear expected prediction rule for regression as

$$
y^{*} \triangleq \mathbf{V}^{\top} \mathbb{E}_{p(\mathbf{h} \mid \mathbf{x}, \mathbf{z})}[\mathbf{h}],
$$


where $\mathbf{V}$ is a $K$-dim vector. To learn the prediction model V, we need to devise a loss function that integrates the large-margin principle for prediction with latent subspace discovery. Here, for prediction, we choose to minimize the $\epsilon$-insensitive loss, which is used in standard support vector regression (SVR) [33]

$$
\mathcal{R}_{\epsilon}(\mathbf{V}) \triangleq \sum_{d} \max \left(0,\left|y_{d}-\mathbf{V}^{\top} \mathbb{E}\left[\mathbf{h}_{d}\right]\right|-\epsilon\right),
$$

where $\epsilon \in \mathbb{R}_{+}$is the precision parameter, which is usually small, and we have defined $\mathbb{E}\left[\mathbf{h}_{d}\right]=\mathbb{E}_{p\left(\mathbf{h} \mid \mathbf{x}_{d}, \mathbf{z}_{d}\right)}[\mathbf{h}]$ for notation simplicity. Similarly, following the regularized risk minimization principle, we learn the entire model for regression and fitting the observed input data by solving the joint optimization problem

$$
\mathrm{P} 2: \min _{\Theta, \mathbf{V}} L(\Theta)+\frac{1}{2} C_{1}\|\mathbf{V}\|_{2}^{2}+C_{2} \mathcal{R}_{\epsilon}(\mathbf{V}),
$$

where $L(\Theta)$ is the negative log-likelihood of input data as we have defined in classification.

Similar to the classification model, by jointly minimizing the negative log-likelihood and the regression loss, we can expect to learn a latent subspace representation as well as a prediction model which on the one hand tend to predict the data accurately, while on the other hand attempt to interpret the data well.

\subsubsection{Optimization with Contrastive Divergence}

Although in principle we can use the similar procedure as in the classification model to solve problem P2 by employing a sub-gradient descent method to learn the parameters $\Theta$, here we use a Lagrangian method to solve an equivalent constrained formulation of P2. One reason is that the loss $\mathcal{R}_{\epsilon}$ is a bit more complicated than $\mathcal{R}_{\text {hinge }}$ because of the non-differentiable absolution operator within the max function. Specifically, problem P2 can be equivalently written as

$$
\begin{aligned}
& \mathrm{P}^{\prime}: \min _{\Theta, \mathbf{V}, \xi, \xi^{\star}} L(\Theta)+\frac{1}{2} C_{1}\|\mathbf{V}\|_{2}^{2}+C_{2} \sum_{d}\left(\xi_{d}+\xi_{d}^{*}\right) \\
& \text { s.t. } \forall d:\left\{\begin{array}{c}
y_{d}-\mathbf{V}^{\top} \mathbb{E}\left[\mathbf{h}_{d}\right] \leqslant \epsilon+\xi_{d} \\
-y_{d}+\mathbf{V}^{\top} \mathbb{E}\left[\mathbf{h}_{d}\right] \leqslant \epsilon+\xi_{d}^{*} \\
\xi_{d}, \xi_{d}^{*} \geqslant 0
\end{array}\right.
\end{aligned}
$$

where $\xi_{d}$ and $\xi_{d}^{*}$ are slack variables.

The constrained problem $\mathrm{P} 2^{\prime}$ is generally intractable because the likelihood $L(\Theta)$ is intractable to evaluate. As in the classification model, we approximate $L(\Theta)$ with the contrastive divergence approximation $\mathcal{L}^{v}\left(q_{0}, q_{1}\right)$. Then, we introduce Lagrange multipliers $\mu_{d}, \mu_{d}^{*}, v_{d}, v_{d}^{*}$ for the four constraints associated with data $d$, and define the Lagrangian function $L$ with the approximate likelihood $\mathcal{L}^{v}\left(q_{0}, q_{1}\right)$

$$
\begin{aligned}
& L=\mathcal{L}^{v}\left(q_{0}, q_{1}\right)+\frac{1}{2} C_{1}\|\mathbf{V}\|_{2}^{2}+C_{2} \sum_{d}\left(\xi_{d}+\xi_{d}^{*}\right)-\sum_{d}\left(v_{d} \xi_{d}+v_{d}^{*} \xi_{d}^{*}\right) \\
& -\sum_{d}\left\{\mu_{d}\left(\epsilon+\xi_{d}-y_{d}+\mathbf{V}^{\top} \mathbb{E}\left[\mathbf{h}_{d}\right]\right)+\mu_{d}^{*}\left(\epsilon+\xi_{d}^{*}+y_{d}-\mathbf{V}^{\top} \mathbb{E}\left[\mathbf{h}_{d}\right]\right)\right\} .
\end{aligned}
$$

Now we optimize the Lagrangian function $L$ by alternatively performing the following steps:
1) Inferring $q_{0}$ and $q_{1}$ : this step is the same as in the classification model.

2) Estimating $\Theta$ with $\mu_{d}$ and $\mu_{d}^{*}$ fixed: this can be solved with gradient descent (e.g., using LBFGS [29] as in the classification model), where the gradients for $(\theta, \eta, \lambda)$ are as before and for $(\mathbf{W}, \mathbf{U})$ we have:

$$
\begin{aligned}
& \partial \mathbf{W}_{e}^{k}=\Delta \mathbb{E}\left[\phi\left(\mathbf{x}_{e}\right) \varphi\left(h_{k}\right)^{\top}\right]-\sum_{d}\left(\mu_{d}-\mu_{d}^{*}\right) \mathbf{V}_{k} \frac{\partial \mathbb{E}_{q_{0}}\left[h_{k}\right]}{\partial \mathbf{W}_{e}^{k}} \\
& \partial \mathbf{U}_{e}^{k}=\Delta \mathbb{E}\left[\psi\left(\mathbf{z}_{e}\right) \varphi\left(h_{k}\right)^{\top}\right]-\sum_{d}\left(\mu_{d}-\mu_{d}^{*}\right) \mathbf{V}_{k} \frac{\partial \mathbb{E}_{q_{0}}\left[h_{k}\right]}{\partial \mathbf{U}_{e}^{k}}
\end{aligned}
$$

3) Estimating the Lagrange multipliers $\left\{\mu_{d}, \mu_{d}^{*}\right\}$ : by setting $\partial L / \partial \xi_{d}, \partial L / \partial \xi_{d}^{*}, \partial L / \partial \mathbf{V}=0$ and exploring the KKT conditions, we can get

$$
\mathbf{V}=\frac{1}{C_{1}} \sum_{d}\left(\mu_{d}-\mu_{d}^{*}\right) \mathbb{E}\left[\mathbf{h}_{d}\right]
$$

Plugging Eq. (12) into the Lagrangian function $L$, we get the dual problem

$$
\begin{aligned}
& \max _{\mu, \mu^{*}}-\frac{1}{2 C_{1}}\left\|\sum_{d}\left(\mu_{d}-\mu_{d}^{*}\right) \mathbb{E}\left[\mathbf{h}_{d}\right]\right\|_{2}^{2}-\sum_{d}\left[\epsilon\left(\mu_{d}+\mu_{d}^{*}\right)-y_{d}\left(\mu_{d}-\mu_{d}^{*}\right)\right] \\
& \text { s.t. } \forall d: \mu_{d}, \mu_{d}^{*} \in\left[0, C_{2}\right],
\end{aligned}
$$

which can be solved using an existing algorithm like SVM-light [25] to obtain $\mu_{d}$ and $\mu_{d}^{*}$.

Again, we can see that in this integrated large-margin formulation for regression, the gradients of $\mathbf{W}$ and $\mathbf{U}$ contain an additional term encoded with $\mu_{d}$ and $\mu_{d}^{*}$. Similar as in the classification model, this additional term introduces a regularization effect to the latent subspace model. If the prediction $\mathbf{V}^{\top} \mathbb{E}\left[\mathbf{h}_{d}\right]$ differs from the true value $y_{d}$ with the absolute gap larger than $\epsilon$, the lagrangian multipliers $\mu_{d}$ or $\mu_{d}^{*}$ (at most one is nonzero because of the KKT conditions) will be non-zero and will bias the model toward discovering a better representation for prediction.

\subsection{Special Case: Maximum Margin Harmonium}

We have developed the large-margin learning framework on a general multi-view latent subspace $\mathrm{MN}$ for classification and regression. In order to fully examine the basic learning principle and compare with existing Harmonium models [41][44][45], we introduce a specialized but very rich instantiation of our supervised latent subspace $\mathrm{MN}$ where the data on each view are not structured. We denote the specialized model by $\mathrm{MMH}$ (max-margin Harmonium). We emphasize that this simplification does not restrict our ability to demonstrate the generability of the framework because both the problem definition and optimization algorithm are general to any structured input data, as we have presented. Specifically, $\mathrm{MMH}$ uses the DWH model detailed at the end of Section 3.1 as the probabilistic likelihood model to fit the input data $(\mathbf{x}, \mathbf{z})$, where $\mathbf{x}$ is a vector of discrete word features (e.g., image tags) and $\mathbf{z}$ is a vector of realvalued features (e.g., color histograms). We can follow the same procedure as above to do parameter estimation. 
For inferring $q_{0}$ and $q_{1}$, the distributions of $\mathbf{x}, \mathbf{z}$ and $\mathbf{h}$ are all fully factorized. Therefore, the sub-gradients in classification or gradients in regression can be easily computed. Details are deferred to Appendix A.1.

\subsection{Time Complexity on Testing}

Before ending this section, we discuss the time complexity of applying the supervised latent subspace MN on various applications, including classification, regression, image retrieval and annotation.

The commonality among using a latent subspace MN for classification, regression and retrieval is that all these applications rely on inferring the latent representations (i.e., $\left.\mathbb{E}_{p(\mathbf{h} \mid \mathbf{x}, \mathbf{z})}[\mathbf{h}]\right)$ only without missing information on the input data. For the large-margin latent subspace $\mathrm{MN}$, since it defines a partial likelihood function, that is, the likelihood on input data $(\mathbf{x}, \mathbf{z})$ only, we can infer these latent representations in a single-round manner. More precisely, the latent representation is $\mathbb{E}_{p(\mathbf{h} \mid \mathbf{x}, \mathbf{z})}[\mathbf{h}]=\Upsilon$, where $\Upsilon_{k}=\sum_{e \in E_{x}} \phi\left(\mathbf{x}_{e}\right)^{\top} \mathbf{W}_{e}^{k}+\sum_{e \in E_{z}} \psi\left(\mathbf{z}_{e}\right)^{\top} \mathbf{U}_{e}^{k}, \forall 1 \leq$ $k \leq K$, which can be very efficiently computed in a linear complexity in terms of the dimensionality of the input features. In contrast, for the MLE-based supervised latent subspace $\mathrm{MN}$, it defines a full likelihood function $p(\mathbf{x}, \mathbf{z}, y)$ over the input data $(\mathbf{x}, \mathbf{z})$ and response value $y$. In testing where $Y$ is not observed, the inference involves an interactive procedure, which iteratively infers the (approximate) posterior distribution of $Y$ and the latent representation. Therefore, the testing time of using the MLE-based supervised latent subspace MN is typically a constant times more expensive than that of the largemargin based method. But in general, these undirected models are much more efficient than their directed counterpart models, as we will show in Section 5.5.

For image annotation, let us use $\mathbf{x}$ to represent tags, which are observed in training. In testing, we infer the posterior distribution $p(\mathbf{x} \mid \mathbf{z})$, which can be approximately computed by running the update Eq. (7) with $\mathbf{z}$ clamped at its observed values. Then, tags with high probabilities are selected as the annotation results. As we can see, the inference procedure is similar as the iterative one of using a MLE-based latent subspace MN for classification. Therefore, the time complexities of the unsupervised and supervised (both large-margin and MLE-based) multi-view MNs are almost the same.

\section{EXPERIMENTS}

Now, we present qualitative as well as quantitative evaluation on three real datasets to demonstrate the advantages (e.g., effectiveness and time efficiency) of largemargin supervised multi-view latent subspace MNs. We first extensively evaluate the specialized but rich $\mathrm{MMH}$ model and compare with extant latent subspace models for classification, regression, image annotation and retrieval in Section 5.3. Then, we present a structured latent subspace $\mathrm{MN}$ for modeling paragraph ordering information on hotel review data in Section 5.4.

\subsection{Datasets and Features}

The datasets ${ }^{6}$ are TRECVID2003 [44], 13class-animal Flickr image data and hotel review data [50]. These datasets are quite rich and diverse in terms of feature types and dimensionality, as detailed below.

TRECVID2003 contains 1078 manually labeled video shots that belong to 5 categories. Each shot is represented as a 1894-dim vector of text features and a 165-dim vector of HSV color histogram, which is extracted from the associated keyframe. We evenly split this dataset into training and testing sets.

The Flickr dataset is a subset selected from NUS-WIDE [12], which is constructed from Flickr web images. This dataset contains 3411 images of 13 animals - squirrel, cow, cat, zebra, tiger, lion, elephant, whales, rabbit, snake, antlers, hawk and wolf. See Fig. 8 for example images from each category. For each image, six types of lowlevel features [12] are extracted, including 634-dim real valued features (i.e., 64-dim color histogram, 144dim color correlogram, 73-dim edge direction histogram, 128-dim wavelet texture and 225-dim block-wise color moments) and 500-dim bag-of-word SIFT [30] features. We randomly select 2054 images for training and use the rest for testing. The 1000-dim online tags are also downloaded for evaluating image annotation.

The hotel review dataset consists of 5000 hotel reviews randomly collected from TripAdvisor ${ }^{7}$. Each review document is associated with two-view features (i.e., 12000-dim bag-of-word features and 14-dim contextual features [50]) as well as a global rating score and five aspect rating scores. The global ratings rank from 1 to 5 . In our experiment, we predict the global rating scores for reviews and uniformly partition the dataset into training and testing sets. Note that the bag-of-words features (e.g., text or SIFT) are treated as binary and modeled using the Bernoulli view.

\subsection{Predictive Latent Subspace Representations}

To demonstrate the power of our method in discovering predictive subspace representations, in this section, we examine various characteristics of the latent subspace representations for modeling both image and text.

\subsubsection{Image Modeling}

We first take a holistic view of the entire latent representations. Fig. 2 shows the 2D embedding of the discovered 10-dim latent representations by MMH, DWH and TWH on the video keyframes in the TRECVID dataset. Here, we use the t-SNE stochastic neighborhood embedding algorithm [36] to embed the latent representations in a 2D space. The results clearly show that the latent subspace representations discovered by $\mathrm{MMH}$ exhibit a strong grouping pattern for the images belonging to the same category, while images from different categories tend

6. http://www.cs.cmu.edu/ junzhu/data.htm

7. http://www.tripadvisor.com 

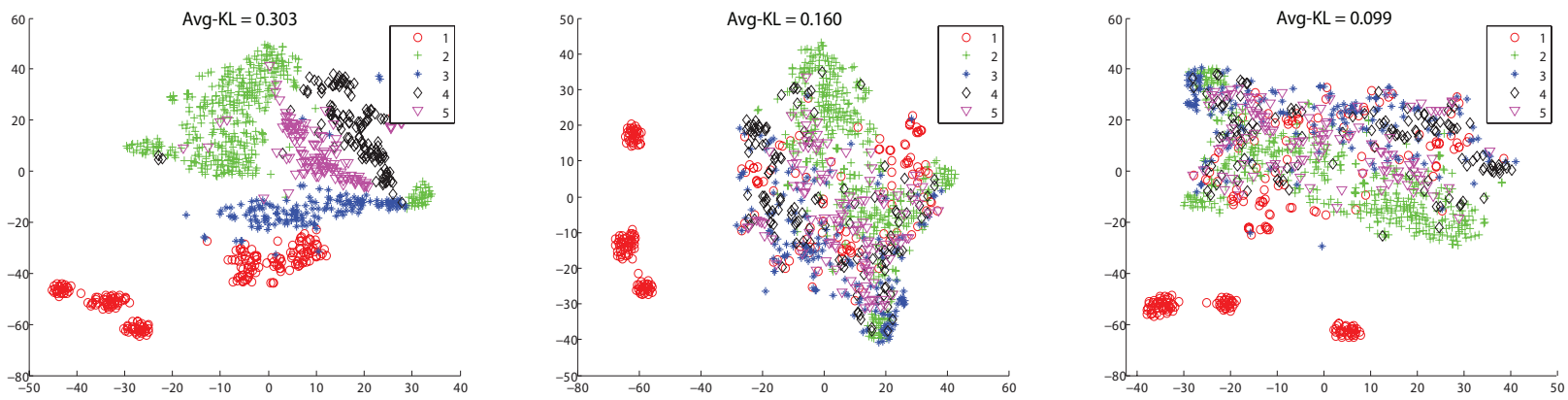

Fig. 2. t-SNE 2D embedding of the discovered latent subspace representation by (Left) MMH, (Middle) DWH and (Right) TWH on the TRECVID video dataset (Better viewed in color).

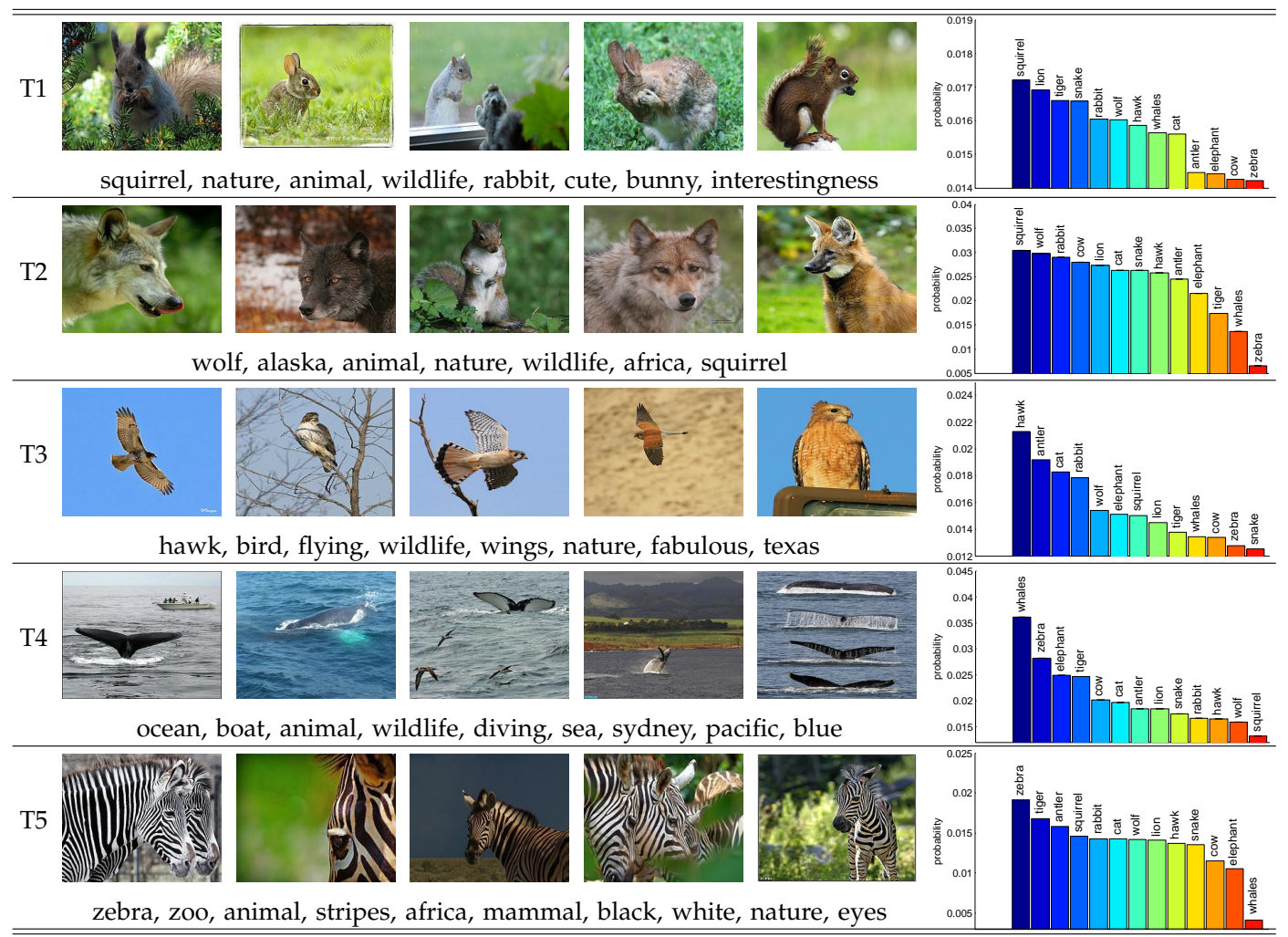

Fig. 3. Example topics discovered by a 60 -topic $\mathrm{MMH}$ on the Flickr animal dataset. For each topic, we show 5 topic-ranked images as well as the average probabilities of that topic on representing images from the 13 categories.

to be separated from each other on the 2D embedding space. In contrast, the latent subspace representations discovered by the likelihood-based DWH and TWH do not show a clear grouping pattern, except for the first category; and images from different categories tend to mix together. These observations suggest that the largemargin based $\mathrm{MMH}$ can discover more discriminative latent subspace representations, which will result in better prediction performance, as we shall see. We have similar observations on the Flickr dataset.

Now, we take a closer examination of each dimension in the discovered latent subspace. We take the Flickr data as an example. Fig. 3 shows five example topics (each topic corresponds to one dimension in the latent subspace) discovered by the large-margin $\mathrm{MMH}$ on the Flickr image data. Due to space limitation, for each topic $\mathrm{T}_{k}$, we show the 5 top-ranked images that yield a high expected value of $H_{k}$, together with the associated tags. Please see Fig. 11 in Appendix A.3 for the 5 bottom- ranked images for each topic. Also, to qualitatively visualize the discriminative power of each topic among the 13 categories, we show the average probability ${ }^{8}$ of each category distributed on the particular topic, as shown in the right part of Fig. 3. From the results, we can see that many of the discovered topics are predictive for one or several categories. For example, topics T3 and T4 are discriminative in predicting the categories hawk and whales, respectively. Similarly, topics T1 and T5 are good at predicting squirrel and zebra, respectively. We also have

8. To compute the distribution, we first turn the expected value of $\mathbf{H}$ to be non-negative by subtracting each element by the smallest value and then normalize it into a distribution over the $K$ topics. The perclass average is computed by averaging the topic distributions of the images within the same class. Then we show the topic distribution on 13 categories specified by different topic. Note that our transformation (i.e., shift-normalization) doesn't affect our interpretation of the discriminative power, which is visually reflected as the (normalized) difference of the average values between categories. Using the raw values of $\mathbb{E}[\mathbf{H}]$ will produce the similar visualization patterns. 
TABLE 1

Average distributions over the topics for documents with different rating scores by a 5-topic MMH and 5-topic $\mathrm{DWH}$.

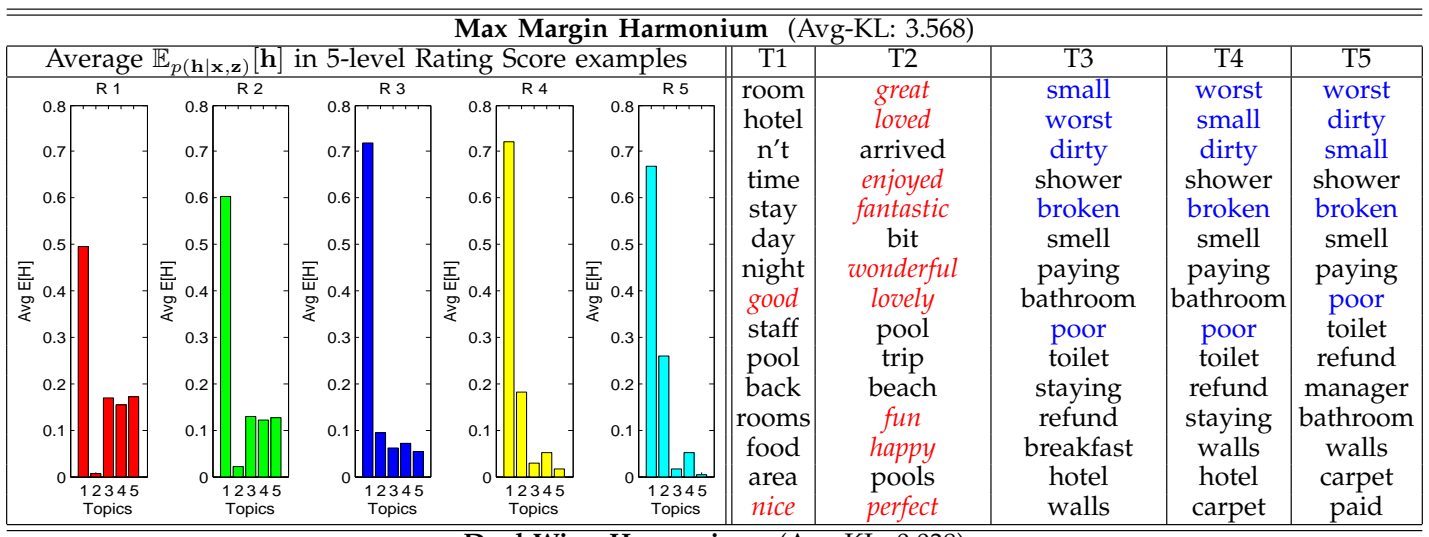

\begin{tabular}{|c|c|c|c|c|c|c|c|c|c|}
\hline \multicolumn{10}{|c|}{ Dual-Wing Harmonium (Avg-KL: 0.038) } \\
\hline Average & $\mathbb{E}_{p(\mathbf{h} \mid \mathbf{x}, \mathbf{z})}[\mathbf{h}$ & in 5-level & Rating Score & examples & T1 & T2 & T3 & T4 & T5 \\
\hline $\mathrm{R} 1$ & R2 & R 3 & R 4 & R5 & room & beach & food & breakfast & belize \\
\hline 0. & 0.5 & 0.9 & 0.9 & 0.9 & hotel & food & told & reception & brett \\
\hline 0.8 & 0.8 & 0.8 & 0.8 & 0.8 & $n^{\prime} t$ & pool & asked & bathroom & cam \\
\hline & & & & & time & resort & holiday & bed & canapes \\
\hline 0.7 & 0.7 & 0.7 & 0.7 & 0.7 & stay & great & reception & shower & canoeing \\
\hline 0.6 & 0.6 & 0.6 & 0.6 & 0.6 & day & restaurants & day & holiday & caracol \\
\hline 至 0.5 & 至 0.5 & 至 0.5 & 至 0.5 & 至 0.5 & night & bar & bar & coffee & hosts \\
\hline 岁 & \begin{tabular}{|ll} 
& 0 \\
& \\
& 0.4
\end{tabular} & 崖 0.4 & 岕 0.0 & 要 0.0 & good & drinks & staff & evening & nadege \\
\hline & & & & & staff & restaurant & back & small & underway \\
\hline 0.3 & 0.3 & 0.3 & 0.3 & 0.3 & pool & lunch & manager & clean & wineries \\
\hline 0.2 & 0.2 & 0.2 & 0.2 & 0.2 & $\begin{array}{l}\text { back } \\
\text { rooms }\end{array}$ & $\begin{array}{c}\text { sea } \\
\text { beautiful }\end{array}$ & $\begin{array}{c}\text { people } \\
\text { evening }\end{array}$ & $\begin{array}{l}\text { bar } \\
\text { hotel }\end{array}$ & $\begin{array}{l}\text { adopted } \\
\text { amanda }\end{array}$ \\
\hline 0.1 & 0.1 & 0.1 . & 0.1 & 0.1 & food & entertainment & entertainment & good & aurora \\
\hline & 0ШП口 & & o & ه & area & pools & arrived & main & begun \\
\hline $\begin{array}{l}12345 \\
\text { Topics }\end{array}$ & $\begin{array}{l}12345 \\
\text { Topics }\end{array}$ & $\begin{array}{l}12345 \\
\text { Topics }\end{array}$ & $\begin{array}{l}12345 \\
\text { Topics }\end{array}$ & $\begin{array}{l}12345 \\
\text { Topics }\end{array}$ & nice & view & hotel & tea & boasted \\
\hline
\end{tabular}

some topics which are good at discriminating a subset of categories against another subset. For example, topic T2 is good at discriminating \{squirrel, wolf, rabbit\} against \{tiger, whales, zebra\}; but it is not very discriminative between squirrel and wolf.

To quantitatively evaluate the predictiveness of the discovered latent subspace representations, we compute the pair-wise average KL-divergence between the perclass average distribution over latent topics ${ }^{9}$. As shown on the top of each plot in Fig. 2, the large-margin based $\mathrm{MMH}$ obtains a larger average KL-divergence score than likelihood-based methods. This again suggests that the latent subspace representations by $\mathrm{MMH}$ are more discriminative or predictive. We obtain similar observations on the Flickr dataset (see Fig. 3 for some example topics), where the average KL-divergence scores of 60-topic MMH, DWH and TWH are 1.62, 1.28 and 0.232 , respectively. This is consistent with our intuitive observations that the latent subspace representations (see Fig. 3) by $\mathrm{MMH}$ are more discriminative.

\subsubsection{Text Modeling}

Now, we examine the properties of latent subspace MN on text modeling. Again, we present both holistic and topic-wise close examinations. Table 1 shows the topics discovered by 5-topic MMH and DWH on the hotel

9. We first turn the expected value of $\mathbf{H}$ into a distribution over the $K$ topics. The per-class average is computed by averaging the topic distributions of the images within the same class. For a pair of distributions $p$ and $q$, the average KL-divergence is $1 / 2(R(p, q)+R(q, p))$. review data. As in [50], we denote the 5 rating scores from small to large by $R 1, R 2, \cdots R 5$. We also show the per-rating average distributions over topics in the left part, which are computed in a similar way as the perclass average distributions in the above section. The right side of Table 1 shows the top 15 words for each topic $\mathrm{T}_{k}$.

Similar to the observations in image modeling, we can see that the latent subspace representations discovered by $\mathrm{MMH}$ are much more discriminative than those discovered by DWH, as reflected from the much higher pair-wise average KL-divergence score and the quite different average distributions over topics, and the individual dimensions (i.e., topics) of the latent subspace learned by MMH are very expressive and discriminative, too. For example, topic T2 for MMH has larger probabilities on representing documents with high rating scores (e.g., $R 5$ and $R 4$ ), but has smaller probabilities (drops to near zero) on documents with lower rating scores (e.g., $R 1$ and $R 2$ ). Moreover, the probability of topic T2 shows a smooth increasing trend on representing documents with rating scores from low to high. If we look at the top words of T2 (e.g., 'great', 'fantastic', 'wonderful', 'perfect', etc.) as shown in the right part of Table 1, we can see that T2 represents a positive aspect of a hotel. Therefore, it is more likely to appear in representing a positive review. In contrast, the negative topics T3, T4 and T5 (e.g., with negative words 'worst', 'dirty', 'poor', etc.) show a smooth decreasing trend on probabilities in representing documents with rating scores from $R 1$ 
to $R 5$. Topic $\mathrm{T} 1$ is kind of neutral which has the highest probability on representing the documents with a neutral rating score (e.g., $R_{3}$ or $R_{4}$ ) and overall $\mathrm{T} 1$ has a much larger probability than any other topics on representing a document. This is reasonable on the hotel review data because most of the words in a review are about the basic hotel information (e.g., 'room', 'hotel', 'food' and 'area'). For DWH, the topics are not very discriminative as demonstrated from the comparable probabilities on representing documents with different rating scores. Table 2 in Appendix A.3 also shows the results on TWH, which is comparable to DWH.

\subsection{Prediction Performance}

In this section, we provide quantitative results on classification, regression, image annotation and retrieval.

\subsubsection{Classification}

We first compare MMH with SVM, DWH, TWH, Gaussian Mixture (GM-Mix), Gaussian Mixture LDA (GMLDA), and Correspondence LDA (CorrLDA) on the TRECVID dataset. See [3] for the details of the last three models. We use SVM ${ }^{\text {multiclass }} 10$ to solve the sub-step of learning $\mathbf{V}$ in $\mathrm{MMH}$ and build the SVM baseline that uses all the available features without distinguishing them in different views. For the unsupervised models (i.e., DWH, GM-Mix, GM-LDA and CorrLDA), a downstream SVM classifier is built based on the discovered latent representations. Fig. 4 (a) shows the classification accuracy of different models, where CorrLDA is omitted because of its too low performance. We can see that the max-margin based multi-view MMH performs consistently better than any other competitors. In contrast, the MLE-based TWH does not show any conclusive improvements compared to the unsupervised DWH. If we train a downstream SVM classifier using the representations by TWH, the classification performance (denoted by $\mathrm{TWH}+\mathrm{SVM}^{11}$ ) will be improved, but still inferior to that of MMH. These results show that supervising side information can help in discovering predictive latent subspace representations that are more suitable for prediction if the model is appropriately learned, e.g., using the large-margin method. The superior performance of MMH compared to the flat SVM demonstrates the usefulness of modeling multi-view inputs for prediction. The reasons for the inferior performance of other models (e.g., CorrLDA and GM-Mix) are analyzed in [44][45].

Fig. 4 (b) shows the accuracy on the Flickr dataset. For brevity, we compare MMH with the best performed DWH, TWH and SVM. We use the 500-dim SIFT and 634-dim real features as two views of inputs for $\mathrm{MMH}$, DWH and TWH. Also, we compare with the single-view

10. http://svmlight.joachims.org/svm_multiclass.html

11. This naïve combination uses supervision twice and is not an elegant model, compared to MMH. The similar naïve combination $\mathrm{MMH}+\mathrm{SVM}$ wouldn't outperform MMH in theory because both methods build SVM classifiers using the same latent representations. See [48] for similar studies.

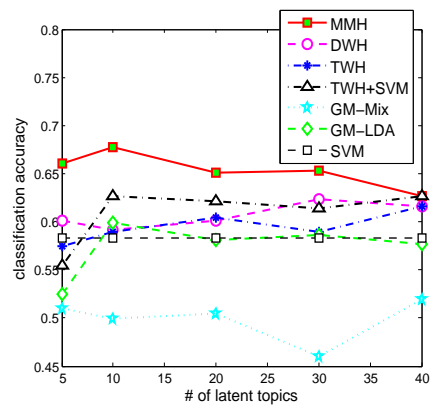

(a)

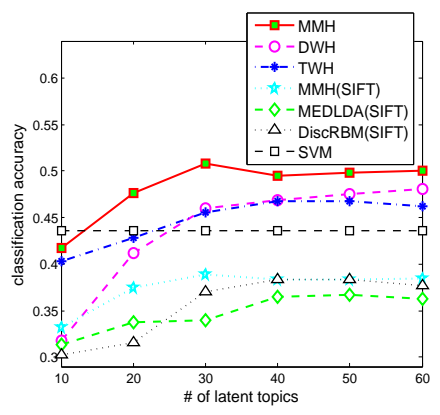

(b)
Fig. 4. Classification accuracy on the (a) TRECVID and (b) Flickr image datasets.

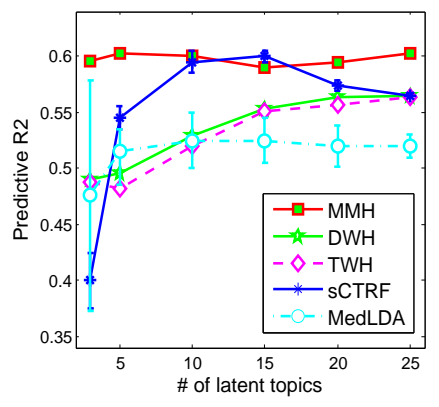

(a)

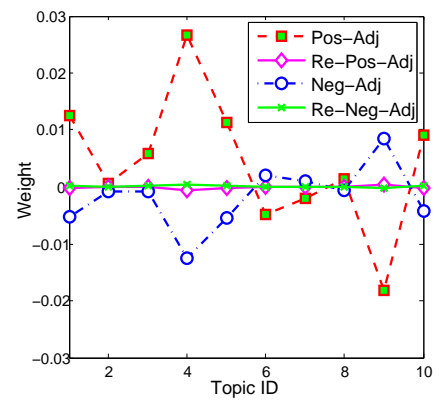

(b)
Fig. 5. (a) Prediction R2; (b) Feature weights in MMH with 10 topics.

MedLDA [48] and discriminative restricted Boltzmann machine (DiscRBM) [28], which use SIFT features only. To be fair, we also evaluate a version of $\mathrm{MMH}$ that uses SIFT features, and denote it by MMH (SIFT). Again, we can see that the large-margin based multi-view MMH performs much better than any other methods, including SVM which ignores the presence of multi-view features. For the single-view MMH (SIFT), it performs comparably with DiscRBM and the large-margin MedLDA, which is a directed $\mathrm{BN}$. As we have stated, $\mathrm{MMH}$ represents an important extension of MedLDA to the undirected latent subspace models and for multi-view data analysis. For DiscRBM, since it performs discriminative training (i.e., maximizing the conditional likelihood of $Y$ given input features) and doesn't estimate the model for generating input features, it can't perform view-level analysis (e.g., predicting image tags). In [28], a hybrid generative/discriminative likelihood objective was also discussed to learn RBM, which outperforms DiscRBM. $\mathrm{MMH}$ is different from the hybrid method in three aspects: 1) our generative likelihood $L(\Theta)$ doesn't include $Y$; 2) our discriminative part is a hinge-loss instead of a conditional log-likelihood; and 3) we use an explicit regularization instead of the implicit regularization (i.e., early stopping) used in [28].

\subsubsection{Regression}

Following [50], we treat the problem of predicting rating scores on hotel review dataset as a regression problem. We compare the MMH regression model with $\mathrm{DWH}$, TWH, sCTRF (i.e., supervised conditional topic random fields) [50] and MedLDA [48]. For DWH, we build a 


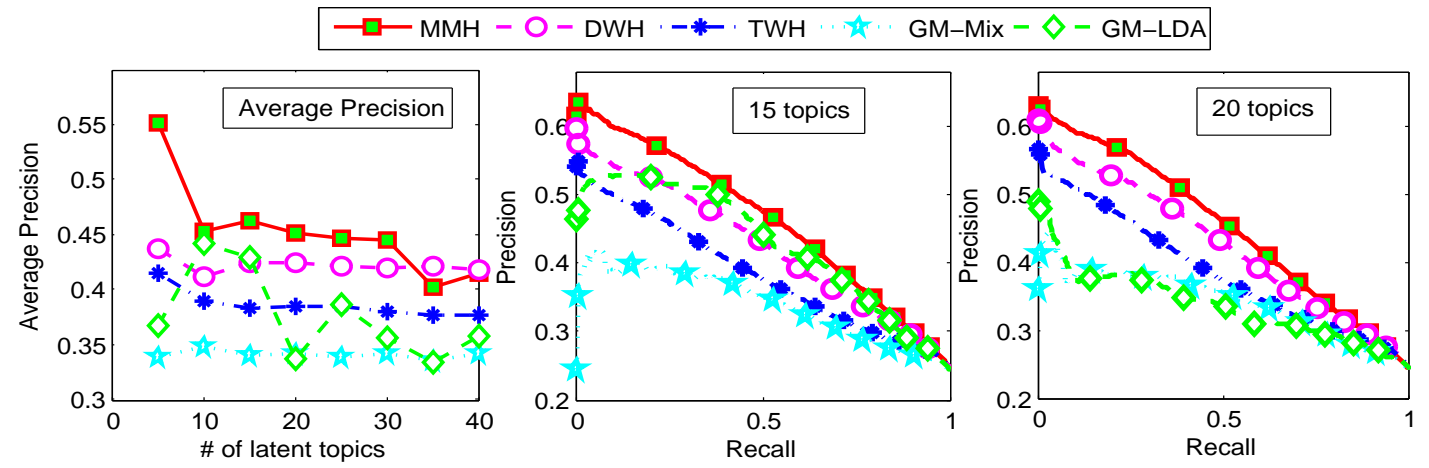

Fig. 6. The average precision curve and the two precision-recall curves for image retrieval on TRECVID data.

linear SVR as the downstream regression model. Many other baselines (e.g., supervised LDA) are not included because they are inferior to sCTRFs as reported in [50]. We consider two views for each document, where one view $\mathbf{X}$ denotes the bag-of-word features and the other view $\mathbf{Z}$ represents the 14 types of contextual features [50].

Fig. 5 (a) shows the predictive R2 scores (please see [4] for the definition). We can observe that by exploring supervising side information (i.e., rating score) in learning the latent subspace model, MMH consistently outperforms the decoupled two-step procedure that is adopted in unsupervised DWH; but the MLE-based TWH does not show improvements over the unsupervised DWH. This again verifies that the large-margin learning plays a significant role in discovering predictive latent subspace representations that are suitable for prediction tasks (e.g., regression). In addition, the reason why $\mathrm{MMH}$ achieves superior performance than the single-view MedLDA is that $\mathrm{MMH}$ can use multi-view features simultaneously, which again demonstrates the benefits of modeling multi-view instead of single-view input for prediction. In fact, the second-view features play an important role in finding a predictive latent subspace. We show the weights of four features on the ten topics discovered by a 10-topic MMH in Fig. 5 (b), where the four features as studied in [50] are: 'Pos-Adj' - positive adjective; 'RePos-Adj' - positive adjective that has a denying word before it, 'Neg-Adj' - negative adjective; and 'Re-NegAdj' - negative adjective that has a denying word before it, respectively. We can see that both the positive and negative adjective features tend to discover topics that are more discriminative for rating prediction (e.g., T4 and T9). The best performance of MMH is comparable to that of SCTRF, which is a directed model. As we shall see in Section 5.5, MMH is much more efficient in training and testing.

\subsubsection{Image Retrieval}

We apply MMH for image retrieval on TRECVID and Flickr datasets. Each test image is a query and training images are ranked based on their cosine similarity ${ }^{12}$ with the given query, which is computed based on the inferred latent subspace representations using the

12. The cosine similarity between vectors $\mathbf{x}_{1}$ and $\mathbf{x}_{2}$ is $\frac{\mathbf{x}_{1}^{\top} \mathbf{x}_{2}}{\left\|\mathbf{x}_{1}\right\|_{2}\left\|\mathbf{x}_{2}\right\|_{2}}$. learned models. An image is considered relevant to the query if they belong to the same category. We evaluate the performance by drawing precision-recall curves and computing the average precision (AP) score [44][45].

Fig. 6 compares MMH with four other models when the topic number $K$ changes. Here, we show the precision-recall curves when $K$ is set at 15 and 20 . Interestingly, although $\mathrm{MMH}$ does not directly optimize a ranking-based loss measure, the latent representation$\mathrm{s}$ discovered by $\mathrm{MMH}$ can result in higher retrieval performance than all other methods in most cases. On the Flickr dataset, we have similar observations. For instance, the AP scores of the 60-topic $\mathrm{MMH}, \mathrm{DWH}$, and TWH are $0.163,0.153$ and 0.158 , respectively.

\subsubsection{Image Annotation}

We also report the annotation results on the Flickr dataset, with a dictionary of 1000 unique tags. The average number of tags per image is about 4.5 . We compare $\mathrm{MMH}$ with DWH and TWH with two views $\mathbf{X}$ for tag and $\mathbf{Z}$ for all the 634-dim realvalued features. We also compare with the sLDA annotation model [39], which uses SIFT features. We use the top- $N$ F1-measure [39], denoted by $F 1 @ N$. With 60 latent topics, the top- $N$ F-measure scores are shown in Fig. 7. Again, we can see that although not directly minimizing an annotation loss measure, the large-margin $\mathrm{MMH}$ outperforms other competitors, mainly because of its good latent representations. Fig. 8 shows example images from all the 13 categories, where for each category the left image is generally of a good annotation quality and the right one is relatively worse.

\subsection{Structured Latent Subspace MN on Modeling Paragraph Ordering Information}

We have extensively evaluated the advantages of largemargin learning based on a specialized dual-wing model (i.e., $\mathrm{MMH}$ ). In this section, we present a structured latent subspace $\mathrm{MN}$ for modeling paragraph ordering information on hotel review data. As we mentioned, 


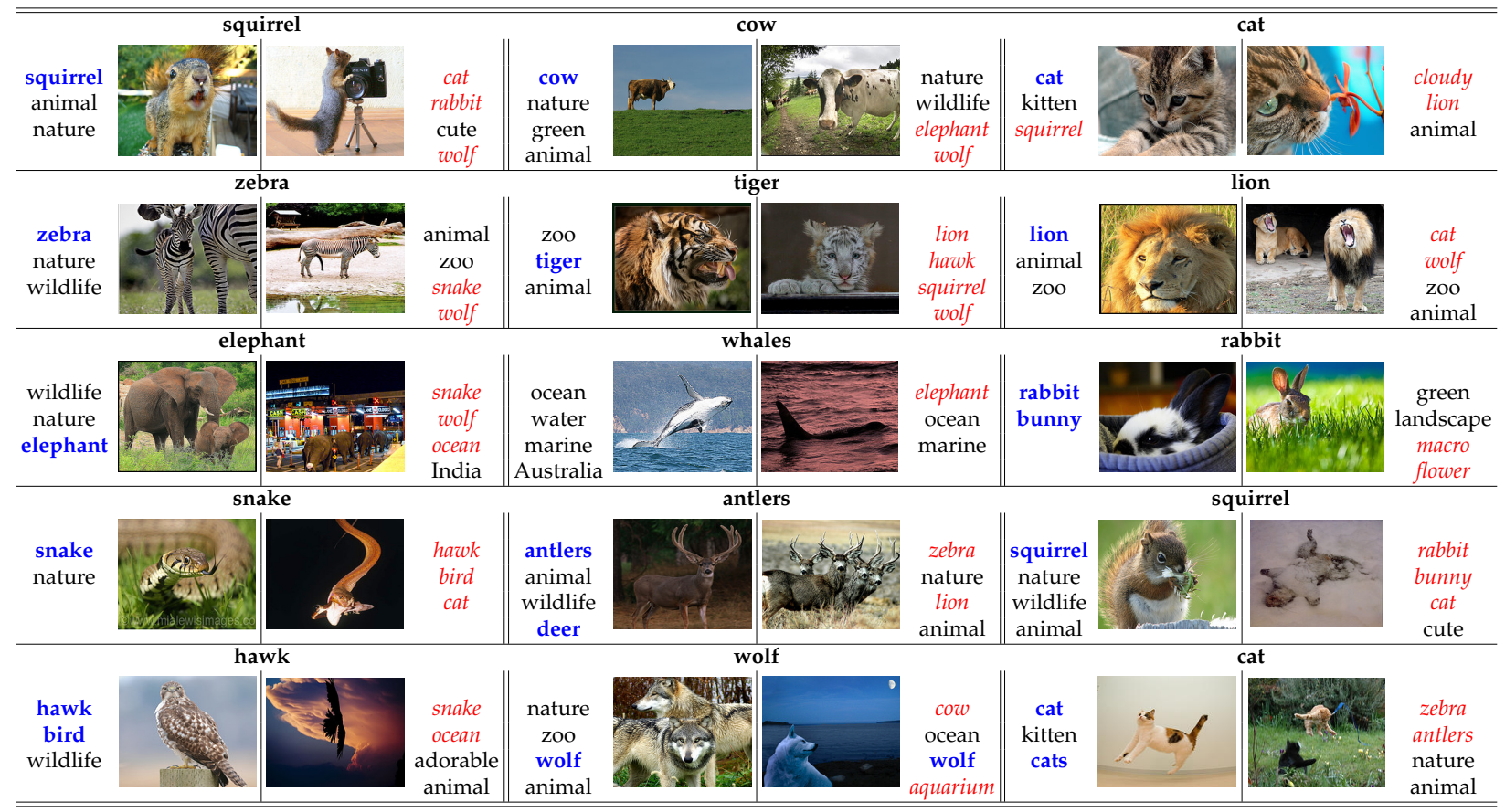

Fig. 8. Example images from the 13 categories on the Flickr animal dataset with predicted annotations. Tags in blue and bold are correct annotations while red and italic ones are wrong predictions. The other tags are neutral. We have repeated the categories "squirrel" and "cat" at the right corner to fill the empty space.

on TripAdvisor there are five pre-defined aspects (e.g., Location), which could guide the users to compose their review contents. Since these aspects are displayed in a particular order to users, we can expect that the composed contents about each aspect would present a similar ordering regularity. Although other possible treatments (e.g., sentence-level ordering) exist, we consider such ordering information between paragraphs and design the structured latent subspace $\mathrm{MN}$ as follows.

We represent a document as a $P \times N$ observation matrix $\mathbf{x}$, where $P$ is the number of paragraphs in this document and $N$ is the vocabulary size. Each row $\mathbf{x}_{p}$ is a vector, of which each element $x_{p i}$ denotes whether word $i$ appears in paragraph $p$. Each column $\mathbf{x}_{. i}$ represents the appearance pattern of word $i$ in all paragraphs. To consider the paragraph ordering information, we define a first-order Markov chain on each $\mathbf{x}_{. i}$ while assuming that different $\mathbf{x}_{. i}$ 's are conditional independent. More formally, we define the conditional distribution $p(\mathbf{x} \mid \mathbf{h})=\prod_{i=1}^{N} p\left(\mathbf{x}_{. i} \mid \mathbf{h}\right)$, where each $p\left(\mathbf{x}_{. i} \mid \mathbf{h}\right)$ is a linear chain CRF [27]. For this structured model, which is in fact an $N$-view latent subspace Markov network, we can perform efficient inference with message passing, whose complexity is also linear in terms of $N$. The details are deferred to Appendix A.2.

To evaluate the structured model, denoted by struct$M M H$, we build another dataset from the hotel reviews on TripAdvisor, which contains 600 reviews for each of the 5 rating scores. We randomly choose a half as training and test on the rest. The reason why we didn't use the dataset [50] is that it contains many reviews that have only one paragraph. Here, while regression can be performed too, we report the classification accuracy in
Fig. 9 (a). We observe that the large-margin structMMH outperforms the unstructured $\mathrm{MMH}$ and the two-stage method (denoted by structDWH) that uses a structured $\mathrm{MN}$ as defined above to infer the latent representations and learn a downstream SVM for classification. This observation demonstrates that the paragraph ordering information is helpful to discover more predictive latent subspace representations for the hotel review data.

\subsection{Running Time and Sensitivity Analysis}

Fig. 9 (b) compares the time efficiency of $\mathrm{MMH}$ with TWH and directed models, including MedLDA and sCTRF [50], on the hotel review dataset [50] for regression. For testing, we can see that: 1) the undirected $\mathrm{MMH}$ and TWH are much more efficient than the directed MedLDA which requires a relatively expensive iterative procedure to infer the distributions of latent variables; 2 ) TWH is about several times slower than MMH because of the reasons as we have discussed in Section 4.4; and 3) SCTRF is about 10 times slower than MedLDA or about 10,000 times slower than $\mathrm{MMH}$. The main reason for such slowness is that SCTRF models every sentence in a document using a Markov chain. Therefore, it spends most of the time on performing message-passing. See [50] for more details. For training, we can see that MMH takes comparable time as TWH and MedLDA, and is much more efficient than SCTRF, whose inference is much slower as shown in testing times.

Finally, as shown in Fig. 10, MMH is not very sensitive to the regularization constant $C_{2}$ on either TRECVID or Flickr dataset when the topic number $K$ is set appropriately. In all the above experiments, we fixed $C_{1}$ at 0.5 and chose $C_{2}$ using cross-validation during training. 


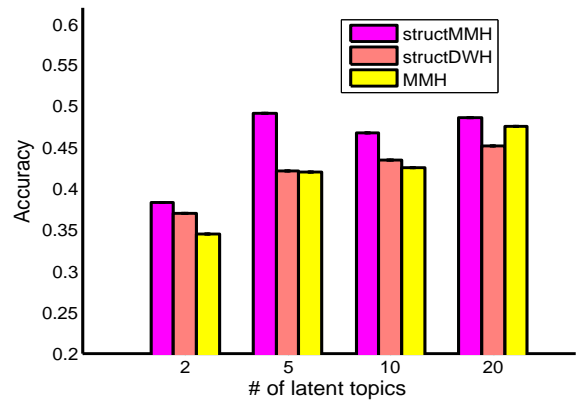

(a)
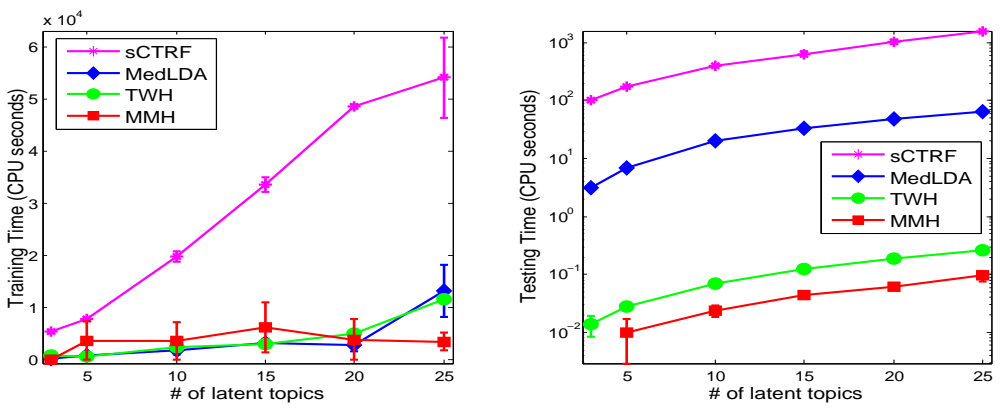

(b)

Fig. 9. (a) classification accuracy of structured $\mathrm{MMH}$ and $\mathrm{DWH}$ models; and (b) training and testing time on hotel review data [50] for regression.

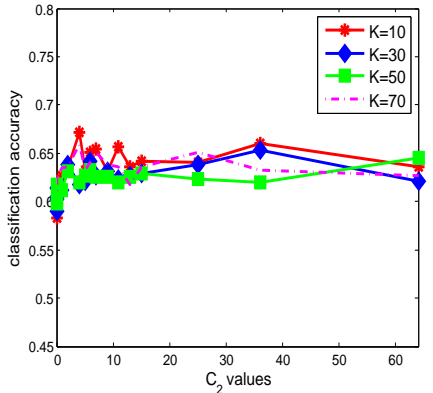

(a)

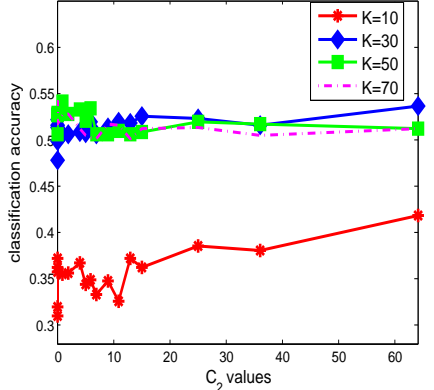

(b)
Fig. 10. Sensitivity to $C_{2}$ on (a) TRECVID and (b) Flickr datasets.

\section{Conclusions AND Discussions}

We have presented a large-margin learning framework for discovering predictive latent subspace representations shared by multi-view data. Besides the proposed multi-view latent subspace Markov networks, the largemargin learning method is generally applicable for the broad family of undirected latent subspace models. The inference and learning can be efficiently done with contrastive divergence methods. Finally, we present extensive evaluation results on various types of real datasets including both image and text data to demonstrate the advantages of large-margin learning for both predictive latent subspace discovery and prediction.

Compared to directed topic models, one drawback of undirected latent subspace models is that their interpretation is generally hard because of the unidentifiability issue [41]. Although our transformation retains the discriminative power, more elegant methods (e.g., imposing non-negative constraints on parameter weights) are needed to improve the interpretability. Another potential limitation of such latent subspace models is that they do not have an explicit control on the sparsity of the discovered latent representations. Sparsity is desirable for large-scale applications where the dimensionality of the latent representations can be tens of thousands. We plan to do systematical studies along these lines. We are also interested in large-scale image annotation and classification, as motivated by the very exciting work [42], where dealing with noisy labeling information is important and challenging in order to learn a robust large-margin model. Finally, we plan to perform more investigation of the large-margin learning method on structured multi-view data analysis, e.g., on text mining [38] and computer vision [22] applications.

\section{ACKNOWLEDGMENTS}

We thank the reviewers for their helpful comments. This work was done at $\mathrm{CMU}$ while $\mathrm{N}$. C. was a visiting researcher there under a CSC fellowship from China. N. C, J. Z. and F.C. S. are supported by a Starting Research Fund from Tsinghua University, No. 553420003, National Key Project for Basic Research of China (Grant No. 2012CB316301, G2007CB311003) and the Tsinghua Self-innovation Project (Grant No:20111081111). J.Z. was supported by ONR N000140910758 at CMU. E.P. X. is supported by ONR N000140910758, NSF IIS-0713379, NSF Career DBI-0546594, and an Alfred P. Sloan Research Fellowship.

\section{REFERENCES}

[1] S. Akaho. A kernel method for canonical correlation analysis. In International Meeting on Psychometric Society, 2001.

[2] K. Ando and T. Zhang. Two-view feature generation model for semi-supervised learning. In International Conference on Machine Learning, 2007.

[3] D. M. Blei and M. I. Jordan. Modeling annotated data. In ACM International Conference on Information Retrieval, pages 127-134, 2003.

[4] D. M. Blei and J. D. McAuliffe. Supervised topic models. In Advances in Neural Information Processing Systems, 2007.

[5] D. M. Blei, A. Y. Ng, and M. I. Jordan. Latent Dirichlet allocation. Journal of Machine Learning Research, 3:993-1022, 2003.

[6] A. Blum and T. Mitchell. Combining labeled and unlabeled data with co-trainnig. In Annual Conference on Learning Theory, 1998.

[7] U. Brefeld and T. Scheffer. Co-EM support vector learning. In International Conference on Machine Learning, 2004.

[8] C. J.C. Burges. A tutorial on support vector machines for pattern recognition. Data Mining and Knowledge Discovery, 2(2):121C-167, 1998.

[9] K. Chaudhuri, S. M. Kakade, K. Livescu, and K. Sridharan. Multiview clustering via canonical correlation analysis. In International Conference on Machine Learning, 2009.

[10] N. Chen, J. Zhu, and E. P. Xing. Predictive subspace learning for multi-view data: a large margin approach. In Advances in Neural Information Processing Systems, 2010.

[11] C. M. Christoudias, R. Urtasun, and T. Darrell. Multi-view learning in the presence of view disagreement. In Conference on Uncertainty in Artificial Intelligence, 2008.

[12] T.-S. Chua, J. Tang, R. Hong, H. Li, Z. Luo, and Y. T. Zheng. NUS-WIDE: A real-world web image database from national university of singapore. In International Conference on Image and Video Retrieval, 2009.

[13] K. Crammer and Y. Singer. On the algorithmic implementation of multiclass kernel-based vector machines. Journal of Machine Learning Research, (2):265-292, 2001. 
[14] M. Culp, G. Michailidis, and K. Johnson. On multi-view learning with additive models. Annals of Applied Statistics, 3(1):292-318, 2009.

[15] T. Diethe, D. R. Hardoon, and J. Shawe-Taylor. Multiview Fisher discriminant analysis. In NIPS Workshop on Learning from Multiple Sources, 2008.

[16] P. Felzenszwalb, R. Girshick, D. McAllester, and D. Ramanan. Object detection with discriminatively trained part based models. IEEE Transactions on Pattern Analysis and Machine Intelligence, 32(9), 2010.

[17] V. Ferrari, T. Tuytelaars, and L. V. Gool. Integrating multiple model views for object recognition. In IEEE Conference on Computer Vision and Pattern Recognition, 2004.

[18] D. Foster, S. Kakade, and T. Zhang. Multi-view dimensionality reduction via canonical correlation analysis. Technical report, Technical Report TR-2008-4, TTI-Chicago, 2008.

[19] Y. Freund. An adaptive version of the boost by majority algorithm. Machine Learning, 43(3):293-318, 2001.

[20] K. Fukumizu, F. Bach, and M. Jordan. Dimensionality reduction for supervised learning with reproducing kernel Hilbert spaces. Journal of Machine Learning Research, (5):73-99, 2004.

[21] K. Ganchev, J. V. Graca, J. Blitzer, and B. Taskar. Multi-view learning over structured and non-identical outputs. In Conference on Uncertainty in Artificial Intelligence, 2008.

[22] D. Gökalp and S. Aksoy. Scene classification using bag-of-regions representations. In IEEE Conference on Computer Vision and Pattern Recognition, 2007.

[23] G. E. Hinton. Training products of experts by minimizing contrastive divergence. Neural Computation, 14(8):1771-1800, 2002.

[24] H. Hotelling. Relations between two sets of variates. Biometrika, 28(3/4):321-377, 1936.

[25] T. Joachims. Making large-scale SVM learning practical. Advances in kernel methods-support vector learning, pages 169-184, 1999.

[26] S. M. Kakade and D. P. Foster. Multi-view regression via conanical correlation analysis. In Annual Conference on Learning Theory, 2007.

[27] J. Lafferty, A. McCallum, and F. Pereira. Conditional random fields: Probabilistic models for segmenting and labeling sequence data. In International Conference on Machine Learning, 2001.

[28] H. Larochelle and Y. Bengio. Classification using discriminative restricted Boltzmann machines. In ICML, 2008.

[29] D. Liu and J. Nocedal. On the limited memory BFGS method for large scale optimization. Mathematical Programming, 45:503-528, 1989.

[30] D. G. Lowe. Object recognition from local scale-invariant features. In IEEE Conference on Computer Vision and Pattern Recognition, 1999.

[31] A. McCallum, C. Pal, G. Druck, and X. Wang. Multi-conditional learning: Generative/discriminative training for clustering and classification. In $A A A I, 2006$.

[32] R. Salakhutdinov and G. E. Hinton. Replicated softmax: an undirected topic model. In Advances in Neural Information Processing Systems, 2009.

[33] A. J. Smola and B. Scholkopf. A tutorial on support vector regression. Statistics and Computing, 14(3):199-222, 2003.

[34] A. Thomas, V. Ferrari, B. Leibe, T. Tuytelaars, B. Schiele, and L. Van Gool. Towards multi-view object class detection. In IEEE Conference on Computer Vision and Pattern Recognition, 2006.

[35] A. Torralba, K. P. Murphy, and W. T. Freeman. Sharing visual features for multiclass and multiview object detection. In IEEE Trans. on Pattern Analysis and Machine Intelligence, 2007.

[36] L. van der Maaten and G. E. Hinton. Visualizing data using tSNE. Journal of Machine Learning Research, 9:2579-2605, 2008.

[37] M. J. Wainwright and M. I. Jordan. Graphical models, exponential families, and variational inference. Foundations and Trends in Machine Learning, 1(1-2):1-305, 2008.

[38] H. M. Wallach. Topic modeling: Beyond bag-of-words. In International Conference on Machine Learning, 2006.

[39] C. Wang, D. M. Blei, and L. Fei-Fei. Simultaneous image classification and annotation. In IEEE Conference on Computer Vision and Pattern Recognition, 2009.

[40] M. Welling and G. E. Hinton. A new learning algorithm for mean field Boltzmann machines. In International Conference on Artificial Neural Networks, 2001.

[41] M. Welling, M. Rosen-Zvi, and G. E. Hinton. Exponential family Harmoniums with an application to information retrieval. In Advances in Neural Information Processing Systems, pages 14811488,2004
[42] J. Weston, S. Bengio, and N. Usunier. Large scale image annotation: Learning to rank with joint word-image embeddings. In European Conference on Machine Learning, 2010.

[43] E. P. Xing, M. I. Jordan, and S. Russell. A generalized mean field algorithm for variational inference in exponential families. In Conference on Uncertainty in Artificial Intelligence, 2003.

[44] E. P. Xing, R. Yan, and A. G. Hauptmann. Mining associated text and images with dual-wing Harmoniums. In Conference on Uncertainty in Artificial Intelligence, 2005.

[45] J. Yang, Y. Liu, E. P. Xing, and A. G. Hauptmann. Harmonium models for semantic video representation and classification. In SIAM Conference on Data Mining, 2007.

[46] C. Yu and T. Joachims. Learning structural SVMs with latent variables. In International Conference on Machine Learning, 2009.

[47] J. Zhang, Z. Ghahramani, and Y. Yang. Flexible latent variable models for multi-task learning. Machine Learning, 73(3):221-242, 2008.

[48] J. Zhu, A. Ahmed, and E. P. Xing. MedLDA: Maximum margin supervised topic models for regression and classification. In International Conference on Machine Learning, 2009.

[49] J. Zhu, L. Li, L. Feifei, and E. P. Xing. Large margin training of upstream scene understanding models. In Advances in Neural Information Processing Systems, 2010.

[50] J. Zhu and E. P. Xing. Conditional topic random fields. In International Conference on Machine Learning, 2010.

[51] J. Zhu, E. P. Xing, and B. Zhang. Partially observed maximum entropy discrimination Markov networks. In Advances in Neural Information Processing Systems, pages 1977-1984, 2008.

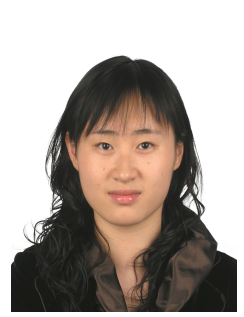

Ning Chen received her BS from China Northwestern Polytechnical University and she is currently working toward her PhD degree in the Department of Computer Science and Technology at Tsinghua University, China. She was a visiting student in Machine Learning Department of Carnegie Mellon University. Her research interests are primarily on probabilistic graphical models, Bayesian Nonparametrics with applications on data mining.

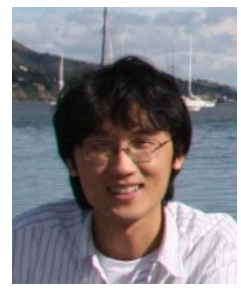

Jun Zhu received his $B S, M S$ and $P h D$ degrees all from the Department of Computer Science and Technology (CS\&T) in Tsinghua University, China, where he is currently an associate professor. He was a project scientist and postdoctoral fellow in the Machine Learning Department, Carnegie Mellon University. His research interests are primarily on developing statistical machine learning methods to understand scientific and engineering data arising from various fields. $\mathrm{He}$ is a member of the IEEE.

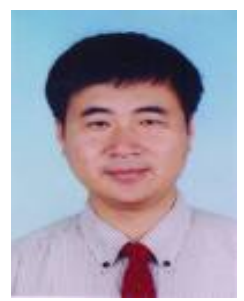

Fuchun Sun received his BS and MS degrees from China Naval Aeronautical Engineering Academy and $\mathrm{PhD}$ degree from the Department of Computer Science and Technology, Tsinghua University. $\mathrm{He}$ is currently a Professor in the Department of Computer Science and Technology, Tsinghua University. His research interests include neural-fuzzy systems, variable structure control, networked control systems, and robotic$\mathrm{s}$. He is a senior member of the IEEE.

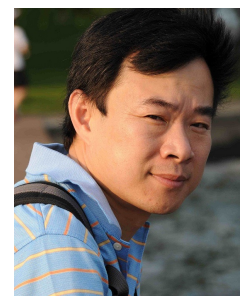

Eric P. Xing received his BS from Tsinghua University, China. He obtained a PhD in Molecular Biology from Rutgers University, and another $\mathrm{PhD}$ in Computer Science from UC Berkeley. He is currently an associate professor in the School of Computer Science at Carnegie Mellon University. His research interests lie in the development of machine learning and statistical methodology; especially for solving problems involving automated learning, reasoning, and decision-making in high-dimensional, multimodal, and dynamic possible worlds in social and biological systems. He is a senior member of the IEEE. 